\title{
Disritmias Cardíacas e Alterações do Segmento ST em Idosos no Perioperatório de Ressecção Transuretral da Próstata sob Raquianestesia. Estudo Comparativo *
}

\section{Cardiac Arrhythmias and ST Changes in the Perioperative Period of Elderly Patients Submitted to Transurethral Prostatectomy Under Spinal Anesthesia. Comparative Study}

\author{
Beatriz Lemos da Silva Mandim, TSA ${ }^{1}$; Renato Enrique Sologuren Achá ${ }^{2}$;
} Neuber Martins Fonseca, TSA ${ }^{3}$; Fabiano Zumpano ${ }^{4}$

\begin{abstract}
RESUMO
Mandim BLS, Achá RES, Fonseca NM, Zumpano F - Disritmias Cardíacas e Alterações do Segmento ST em Idosos no Perioperatório de Ressecção Transuretral da Próstata sob Raquianestesia. Estudo Comparativo
\end{abstract}

JUSTIFICATIVA E OBJETIVOS: Idosos representam 25\% do total dos pacientes cirúrgicos. Muitos pacientes com doença arterial coronariana (DAC) apresentam eletrocardiograma (ECG) pré-operatório normal, e alta incidência de infarto agudo do miocárdio (IAM) silencioso na $1^{\text {a }}$ semana de pós-operatório. As disritmias aumentam com a idade, sendo observadas extrassístoles supraventriculares (ESSV) e ventriculares (ESV), fibrilação atrial e distúrbios da condução intraventricular. O objetivo deste estudo foi avaliar a prevalência de disritmias cardíacas e de alterações do segmento ST no perioperatório através do Sistema Holter em pacientes idosos submetidos à cirurgia de ressecção transuretral da próstata (RTU) e herniorrafia inguinal sob raquianestesia (controle).

MÉTODO: Foram avaliados 21 pacientes com idades entre 65 e 84 anos submetidos a RTU da próstata e 16 pacientes com idades entre 65 e 86 anos, submetidos à herniorrafia inguinal, sob raquianestesia. Avaliação pelo Sistema Holter no pré-operatório (12 horas), intra-operatório (3 horas) e pós-operatório (12 horas).

RESULTADOS: A prevalência de extrassístoles supraventriculares (ESSV) entre os grupos RTU e controle foi, no pré-operatório $85,7 \%$ vs. $93,7 \%$, no intra-operatório $85,7 \%$ vs. $81,2 \%$ e no pós-operatório $76,2 \%$ vs. $100 \%$. As extrassístoles ventriculares (ESV) tiveram prevalência de $76,2 \%$ vs. $81,2 \%$ no pré, $80,9 \%$ vs. $68,7 \%$ no intra e $80,9 \%$ vs. $81,2 \%$ no pós-operatório. A prevalência de alterações do segmento ST entre os grupos RTU e controle foi, no pré-operatório $19 \%$ vs. $18,7 \%$, no intra-operatório $4,7 \%$ vs. $18,7 \%$ e no pós-operatório de $14,3 \%$ vs. 18,7\%, sem significância estatística.

\footnotetext{
* Recebido do (Received from) Departamento de Anestesiologia da Universidade Federal de Uberlândia (UFU), MG

1. Co-Responsável pelo CET/SBA; Professora Substituta Mestre da Disciplina de Anestesiologia da UFU

2. Professor Titular Doutor da Disciplina de Cardiologia da UFU

3. Responsável pelo CET/SBA; Professor Adjunto Doutor da Disciplina de Anestesiologia da UFU

4. EX-ME 2 do CET/SBA da UFU
}

Apresentado (Submitted) em 25 de março de 2003 Aceito (Accepted) para publicação em 17 de junho de 2003

Endereço para correspondência (Correspondence to)

Dra. Beatriz Lemos da Silva Mandim

Rua Berenice Rezende Diniz, 300 - Casa 22 - Gávea

38411-162 Uberlândia, MG

E-mail:mandim@uol.com.br

(c) Sociedade Brasileira de Anestesiologia, 2004
CONCLUSÕES: Os paciente idosos apresentam alta prevalência de ESSV e ESV. O número total de ESSV e ESV, e alterações do segmento $S T$, presentes no período pré-operatório, não foi alterado pela cirurgia de ressecção transuretral da próstata, bem como pela herniorrafia inguinal, nos períodos intra e pós-operatório.

Unitermos: CIRURGIA, Urológica: prostatectomia; COMPLICAÇÕES: disritmias cardíacas; TÉCNICAS ANESTÉSICAS, Regional: subaracnóidea

\section{SUMMARY}

Mandim BLS, Achá RES, Fonseca NM, Zumpano F - Cardiac Arrhythmias and ST Changes in the Perioperative Period of Elderly Patients Submitted to Transurethral Prostatectomy Under Spinal Anesthesia. Comparative Study.

BACKGROUND AND OBJECTIVES: Elderly account for $25 \%$ of surgical patients. Several patients with arterial heart disease have normal preoperative ECG and a high incidence of silent myocardial acute infarction in the first postoperative week. Arrhythmias increase with age and supraventricular and ventricular premature complexes, atrial fibrillation and intraventricular conduction abnormalities are observed. This study aimed at evaluating the prevalence of perioperative arrhythmias and ST changes through Holter System in elderly patients submitted to transurethral prostatectomy and inguinal hernia repair under spinal anesthesia.

METHODS: Participated in this study 21 patients aged 65 to 84 years submitted to transurethral prostatectomy (TUP) and 16 patients aged 63 to 86 years submitted to inguinal hernia repair under spinal anesthesia. Monitoring with Holter System was performed in the preoperative (12 hours), intraoperative (3 hours) and postoperative (12 hours) periods.

RESULTS: The prevalence of supraventricular premature complex was $85.7 \%$ vs. $93.7 \%$ in the preoperative period, $85.7 \%$ vs. $81.2 \%$ in the intraoperative and $76.2 \%$ vs. $100 \%$ in the postoperative period, respectively for groups TUP and control. The prevalence of ventricular premature complex was $76.2 \%$ vs. $81.2 \%$ in the preoperative period, $80.9 \%$ vs. $68.7 \%$ in the intraoperative period, and $80.9 \%$ vs. $81.2 \%$ in the postoperative period, respectively for groups TUP and control. The prevalence of ST changes was $19 \%$ vs. $18.7 \%$ in the preoperative period, $4.7 \%$ vs. $18.7 \%$ in the in the intraoperative period, and $14.3 \%$ vs. $18.7 \%$ in the postoperative period between groups TUP and control, without statistical significance.

CONCLUSIONS: Elderly patients have a high prevalence of supraventricular and ventricular cardiac arrhythmias. The total number of preoperative arrhythmias and ST changes was not changed as a function of transurethral prostatectomy surgery or inguinal hernia repair, in the intra and postoperative periods.

Key Words: ANESTHETIC TECHNIQUES, Regional: spinal block; COMPLICATIONS: cardiac arrhythmias; SURGERY, Urologic: prostatectomy 


\section{INTRODUÇÃO}

$\mathrm{N}^{\mathrm{a}}$ ão existe uma definição precisa para "velho", "idoso" ou "idade avançada", como não há também um marcador clínico específico para o paciente geriátrico, já que o enveIhecimento não ocorre abruptamente, mas se apresenta como um processo contínuo ${ }^{1}$.

A população geriátrica é única por sua heterogeneidade ${ }^{2}$. A Organização Mundial de Saúde, baseada em fatores sócio-econômicos, considera idoso todo indivíduo com 65 anos ou mais. Porém, no Brasil, do ponto de vista legal, idoso é toda pessoa com 60 anos de idade ou mais (Lei 8.842, de janeiro de 1994$)^{3}$. De modo geral, as funções fisiológicas declinam à razão de $1 \%$ ao ano após a idade de 30 anos, sendo que com 70 anos, o metabolismo basal é de $40 \%$ do normal ${ }^{4}$. São relacionadas como doenças prevalentes no pré-operatório de idosos as alterações eletrocardiográficas, a arteriosclerose cardiovascular, o infarto agudo do miocárdio prévio, a insuficiência cardíaca congestiva, a hepatopatia e a nefropatia ${ }^{5}$.

Idosos representam $25 \%$ do total dos pacientes cirúrgicos, e estima-se que $50 \%$ das pessoas com mais de 65 anos sofrerão uma cirurgia antes de morrerem ${ }^{6}$.

Muitos pacientes com doença arterial coronariana (DAC) apresentam eletrocardiograma (ECG) normal antes da cirurgia ${ }^{7}$. Isquemia miocárdica silenciosa (IMS) detectada pela monitorização da eletrocardiografia dinâmica de longa duração (Sistema Holter)é comum durante as atividades diárias em pacientes assintomáticos com doença arterial coronariana e que têm testes de esforço na esteira positivos ${ }^{8}$. Nos pacientes com angina, $80 \%$ dos episódios isquêmicos são silenciosos ${ }^{9}$. Há uma alta incidência de IMS na primeira semana de pós-operatório, variando de $21 \%{ }^{10}$ a $60 \%{ }^{11}$. A primeira suspeita clínica de isquemia perioperatória se apresenta como baixo débito cardíaco ${ }^{11,12}$, hipotensão arterial ou disritmia ${ }^{13}$.

A incidência de alterações eletrocardiográficas em pacientes idosos aumenta com a idade, sendo observadas: alterações da onda T e do segmento ST, extrassístoles supraventriculares (ESSV) e ventriculares (ESV) e fibrilação atrial, distúrbios da condução intraventricular (bloqueio do ramo direito, bloqueio divisional ântero-superior esquerdo e bloqueio do ramo esquerdo) e bloqueio atrioventricular de $1^{\circ} \mathrm{grau}$. Algumas são freqüentes em pacientes idosos (extrassístoles, fibrilação atrial, bloqueio do ramo esquerdo, distúrbio de condução intraventricular e alterações de ST), enquanto outras correlacionam-se melhor com a presença de alterações anatômicas associadas (onda $Q$ patológica, bloqueio do ramo direito e bloqueio divisional ântero-superior esquerdo) ${ }^{14}$. Bertrand e col. ${ }^{15}$ (1971) relataram incidência de $48 \%$ de disritmias durante a recuperação da anestesia geral, sendo $28 \%$ ventriculares.

Àmedida que o indivíduo envelhece, ocorrem mudanças fisiológicas no coração que afetam o miocárdio e o tecido de condução, entre outras. Estas alterações independem das que resultam de processos patológicos, freqüentes no idoso. Diversas alterações na eletrofisiologia cardíaca relacionadas à idade se assemelham às produzidas pelas doenças ${ }^{16}$.
Mudanças estruturais levam a aumento do tecido colágeno e da substância amilóide no miocárdio e redução das células do tecido específico de condução ${ }^{17}$ e também da sensibilidade aos estímulos adrenérgicos ${ }^{18}$.

A cirurgia de ressecção transuretral da próstata (RTU) é realizada na população idosa e associa-se com morbidade considerável. A mortalidade por causas cardiovasculares após RTU é de $0,5 \%$ a $1 \%$, aumentando para mais de $2 \%$ nos pacientes acima de 80 anos ${ }^{19,20}$.

Existem trabalhos avaliando as alterações do segmento ST ou IMS durante a cirurgia de ressecção transuretral da próstata, porém não foram encontrados estudos que verificassem a prevalência das disritmias durante o período intra-operatório, utilizando eletrocardiografia dinâmica (Sistema Holter), em pacientes submetidos a este tipo de cirurgia, sob raquianestesia.

O objetivo deste estudo foi avaliar a prevalência de disritmias cardíacas e de alterações do segmento ST no perioperatório através do Sistema Holter em pacientes idosos submetidos à cirurgia de ressecção transuretral da próstata (RTU) e herniorrafia inguinal sob raquianestesia.

\section{MÉTODO}

Após aprovação pelo Comitê de Ética em Pesquisa da Universidade Federal de Uberlândia, foram estudados aleatoriamente 21 pacientes portadores de hipertrofia benigna da próstata com idade variando de 65 a 84 anos, submetidos a RTU da próstata (grupo RTU), e 16 pacientes portadores de hérnia inguinal unilateral (grupo controle), com idades de 65 a 86 anos e submetidos à herniorrafia inguinal sob raquianestesia, no período de novembro de 2001 a agosto de 2002 . Todos os pacientes foram consultados sobre a participação no projeto, informados sobre as finalidades da pesquisa e assinaram termo de consentimento esclarecido. Foram excluídos do estudo pacientes portadores de sorologia positiva para doença de Chagas, com alterações do segmento ST no eletrocardiograma, portadores de nefropatias, hepatopatias, anemia, infecções e também aqueles que apresentassem contra-indicação para raquianestesia.

Os pacientes foram avaliados na fase pré-operatória por meio de anamnese, exame físico, laboratorial e eletrocardiografia de 12 derivações. Isquemia miocárdica foi definida como horizontalização ou depressão do segmento ST por 1 $\mathrm{mm}$ ou mais ou elevação do segmento ST de $2 \mathrm{~mm}$ ou mais, durando 60 segundos ou mais e seguida por retorno à linha de base por pelo menos 1 minuto ${ }^{21}$.

Realizou-se o eletrocardiograma dinâmico de longa duração (Sistema Holter), usando gravador Dynamics 3000 e analisado pelo programa Cardiosistema, versão ALT V5 08C, nos períodos pré-operatório: 12 horas antes do início da anestesia; intra-operatório: durante todo o procedimento cirúrgico, desde o início da anestesia até o seu término, com duração de 3 horas, baseado na duração do anestésico local bupivacaína, que é de 180 minutos ${ }^{22}$; pós-operatório: 12 horas após o término da anestesia. 
Foram avaliados o ritmo cardíaco; freqüência cardíaca (bradicardia e taquicardia sinusal); presença de disritmias cardíacas, classificação (supraventricular ou ventricular) e complexidade (extrassístoles isoladas, pareadas ou em salvas), presença de bloqueios atrioventriculares e/ou de ramos do feixe de His, pausas e alterações do segmento ST, considerando os períodos pré, intra e pós-operatório.

Os pacientes de ambos os grupos não receberam medicação pré-anestésica. As doses usuais de medicação anti-hipertensiva e cardiovascular, com exceção da aspirina, foram mantidas. Na sala cirúrgica foi estabelecida hidratação venosa, 30 minutos antes de iniciar a anestesia, com $500 \mathrm{ml}$ de solução de Ringer com lactato. A monitorização constou de cardioscópio na derivação CM5, esfigmomanômetro para controle da pressão arterial pelo método Riva-Rocci, com aferição a cada 5 minutos e oximetria de pulso para leitura contínua da saturação de oxigênio $\left(\mathrm{SpO}_{2}\right)$. Foi realizada raquianestesia, com o paciente na posição de decúbito lateral direito e infiltração prévia da pele e subcutâneo com lidocaína a $1 \%$ no local da punção, em $\mathrm{L}_{2} \mathrm{~L}_{3}$ ou $\mathrm{L}_{3-} \mathrm{L}_{4}$, por via mediana, com aguIha tipo Quincke $25 \mathrm{G}$ com bisel cefálico. Após a identificação do espaço subaracnóideo pela presença do líquor cefalorraquidiano, foram administrados 14 mg de bupivacaína hiperbárica a $0,5 \%$.

Imediatamente após a injeção, o paciente foi colocado na posição supina até que o bloqueio, verificado pela perda de sensibilidade ao frio, atingisse o nível $\mathrm{T}_{10}$, quando então o paciente foi colocado na posição cirúrgica. Quando a pressão arterial atingiu um nível de $20 \%$ abaixo do valor inicial, foi administrado o vasopressor efedrina $(10 \mathrm{mg})$. A analgesia pós-operatória foi realizada sistematicamente com dipirona (1 g) por via venosa administrada a cada 6 horas.

ARTU da próstata foi realizada na posição de litotomia, utilizando irrigação contínua de água destilada em temperatura ambiente, com altura de até $50 \mathrm{~cm}$ em relação ao paciente.

Para as variáveis em que as amostras são relacionadas, foi aplicada a prova não-paramétrica de Wilcoxon, para comparar cada grupo no pré, intra e pós-operatório. Para as variáveis em que as amostras são independentes, foi aplicada a prova $U$ de Mann-Whitney, para comparar os dois grupos em cada período analisado.

O teste $t$ de Student foi aplicado entre médias de amostras independentes, quando se comparou idade, prevalência de bradicardia, de disritmias, de alterações do segmento ST e complexidade das disritmias entre os grupos.

Em todos os testes, fixou-se em 0,05 ou $5 \%(p<0,05)$ o nível para a rejeição da hipótese de nulidade, indicando-se com asterisco os valores significantes.

\section{RESULTADOS}

Os grupos foram homogêneos quanto aos dados demográficos e estado físico (Tabela I).

Na avaliação pré-operatória, observou-se que dois pacientes no grupo RTU já haviam apresentado infarto agudo do miocárdio prévio, enquanto, que no grupo controle não havia nenhum, com diferença significante entre eles. Os demais parâmetros avaliados foram semelhantes nos dois grupos, sem diferença significante (Tabela II).

Tabela I - Dados Demográficos

\begin{tabular}{lcc}
\hline Parâmetros & $\begin{array}{c}\text { Grupo RTU } \\
(\mathrm{n}=21)\end{array}$ & $\begin{array}{c}\text { Grupo Controle } \\
(\mathrm{n}=16)\end{array}$ \\
\hline Idade $(\mathrm{anos}){ }^{*}$ & $73,05 \pm 6,79$ & $70,5 \pm 6,11$ \\
Peso $(\mathrm{kg})^{*}$ & $69,24 \pm 13,13$ & $65,13 \pm 10,29$ \\
Altura $(\mathrm{m})^{*}$ & $1,69 \pm 0,04$ & $1,67 \pm 0,07$ \\
Estado Físico & & \\
$\quad$ ASA II & $16(76,19 \%)$ & $14(87,50 \%)$ \\
ASA III & $5(23,81 \%)$ & $2(12,50 \%)$ \\
\hline
\end{tabular}

* Dados expressos pela Média \pm DP. Teste $t$ de Student: $p>0,05$

Tabela II - Avaliação Clínica Pré-Operatória

\begin{tabular}{lcc}
\hline Parâmetros & $\begin{array}{c}\text { Grupo RTU } \\
(\mathrm{n}=21)\end{array}$ & $\begin{array}{c}\text { Grupo Controle } \\
(\mathrm{n}=16)\end{array}$ \\
\hline Infarto agudo do miocárdio & $2(9,52 \%)^{*}$ & $0(0 \%)$ \\
Hipertensão arterial sistêmica & $9(42,86 \%)$ & $8(50 \%)$ \\
Diabetes melito & $1(4,76 \%)$ & $2(12,50 \%)$ \\
Tabagismo & $3(14,28 \%)$ & $2(12,50 \%)$ \\
\hline
\end{tabular}

*Teste $t$ de Student: $p<0,05$

Quanto aos exames pré-operatórios, observamos uniformidade entre os grupos (Tabela III).

Tabela III - Exames Pré-Operatórios

\begin{tabular}{lcc}
\hline Parâmetros & $\begin{array}{c}\text { Grupo RTU } \\
(n=21)\end{array}$ & $\begin{array}{c}\text { Grupo Controle } \\
(n=16)\end{array}$ \\
\hline Hemoglobina * & $13,9 \pm 1,6$ & $15,2 \pm 1,7$ \\
Glicemia * & $111,7 \pm 36,1$ & $115,3 \pm 27,5$ \\
ECG normal & $2(9,5 \%)$ & $4(25 \%)$ \\
ECG com disritmias & $12(57,1 \%)$ & $6(37,5 \%)$ \\
ECG sinais indiretos de & $14(66,7 \%)$ & $7(43,7 \%)$ \\
\hline infarto agudo do miocárdio & &
\end{tabular}

* Dados expressos pela Média \pm DP. Teste $t$ de Student: $p>0,05$

A tabela IV mostra o número de pacientes que apresentaram bradicardia sinusal $(F C<60)$, pausas sinusais e alterações do segmento ST, comparando os grupos RTU e controle no pré, intra e pós-operatório, bem como a média por hora estudada em cada período, não sendo observadas diferenças significantes entre os grupos, exceto pela média de pausas sinusais no intra-operatório do grupo RTU, onde 1 paciente apresentou 107 pausas.

Os resultados observados na tabela $\mathrm{V}$ mostram o número total de extrassístoles supraventriculares e ventriculares nos grupos RTU e controle sendo que, quando comparou-se os períodos de cada grupo e também os dois grupos em cada período, não foram encontradas diferenças significantes.

Revista Brasileira de Anestesiologia Vol. 54, Nº 2, Março - Abril, 2004 
Tabela IV - Bradicardia Sinusal, Pausas Sinusais e Alterações no Segmento ST nos Períodos Pré, Intra e Pós-Operatório do Grupo RTU e Grupo Controle

\begin{tabular}{|c|c|c|c|c|c|c|}
\hline & \multicolumn{2}{|c|}{ Pré-Operatório (12 h) } & \multicolumn{2}{|c|}{ Intra-Operatório (3 h) } & \multicolumn{2}{|c|}{ Pós-Operatório (12 h) } \\
\hline & $\begin{array}{c}\text { RTU } \\
(\mathrm{n}=21)\end{array}$ & $\begin{array}{l}\text { Controle } \\
(n=16)\end{array}$ & $\begin{array}{c}\text { RTU } \\
(\mathrm{n}=21)\end{array}$ & $\begin{array}{l}\text { Controle } \\
(n=16)\end{array}$ & $\begin{array}{c}\text { RTU } \\
(\mathrm{n}=21)\end{array}$ & $\begin{array}{l}\text { Controle } \\
(n=16)\end{array}$ \\
\hline $\mathrm{FC}<60-\mathrm{n}(\%)$ & $18(87 \%)$ & $12(75 \%)$ & $20(95 \%)$ & $11(68,7 \%)$ & $18(86 \%)$ & $11(68,7 \%)$ \\
\hline Média/h $\pm \mathrm{DP}$ & $46 \pm 10,4$ & $52,6 \pm 10,1$ & $43,7 \pm 10,2$ & $52,7 \pm 12,5$ & $46,7 \pm 10,8$ & $54,6 \pm 13,1$ \\
\hline Pausas - n (\%) & $2(9 \%)$ & $1(6,2 \%)$ & $1(5 \%)$ & $1(6,2 \%)$ & $1(5 \%)$ & $2(12,5 \%)$ \\
\hline Média/h \pm DP & $0,003 \pm 0,02$ & $0,03 \pm 0,12$ & $1,7 \pm 7,8^{*}$ & $0,06 \pm 0,25$ & $0,007 \pm 0,04$ & $0,01 \pm 0,03$ \\
\hline \multicolumn{7}{|l|}{ Alterações ST } \\
\hline ST - n (\%) & $4(19 \%)$ & $3(18,7 \%)$ & $1(4,8 \%)$ & $3(18,7 \%)$ & $3(14,3 \%)$ & $3(18,7 \%)$ \\
\hline Média/h $\pm \mathrm{DP}$ & $0,04 \pm 0,11$ & $0,15 \pm 0,34$ & $0,02 \pm 0,07$ & $0,12 \pm 0,3$ & $0,03 \pm 0,08$ & $0,12 \pm 0,25$ \\
\hline
\end{tabular}

* Teste $t$ de Student $p<0,05$

Tabela V - Número Total de Extrassístoles Supraventriculares (ESSV) e Ventriculares (ESV) nos Grupos RTU e Controle

\begin{tabular}{|c|c|c|c|c|c|c|c|c|c|c|c|c|}
\hline & \multicolumn{6}{|c|}{ Extrassístoles Supraventriculares } & \multicolumn{6}{|c|}{ Extrassístoles Ventriculares } \\
\hline & \multicolumn{3}{|c|}{ Grupo RTU } & \multicolumn{3}{|c|}{ Grupo Controle } & \multicolumn{3}{|c|}{ Grupo RTU } & \multicolumn{3}{|c|}{ Grupo Controle } \\
\hline & Pré (12h) & Intra (3h) & Pós (12h) & Pré (12h) & Intra (3h) & Pós (12h) & Pré (12h) & Intra (3h) & Pós (12h) & Pré (12h) & Intra (3h) & Pós (12h) \\
\hline 1 & 63 & 7 & 14 & 54 & 98 & 49 & 0 & 0 & 3 & 2 & 2 & 1 \\
\hline 2 & 2 & 1 & 7 & 20 & 0 & 15 & 29 & 6 & 41 & 5 & 0 & 12 \\
\hline 3 & 94 & 74 & 90 & 0 & 0 & 2 & 0 & 0 & 0 & 110 & 31 & 117 \\
\hline 5 & 4 & 0 & 0 & 8 & 7 & 9 & 0 & 4 & 0 & 1 & 1 & 3 \\
\hline 6 & 335 & 67 & 415 & 445 & 62 & 158 & 1469 & 376 & 1457 & 1173 & 22 & 1344 \\
\hline 7 & 11 & 8 & 23 & 19 & 5 & 66 & 3 & 3 & 6 & 4199 & 290 & 2804 \\
\hline 8 & 128 & 24 & 129 & 34 & 15 & 17 & 94 & 41 & 389 & 30 & 4 & 2 \\
\hline 9 & 31 & 7 & 19 & 90 & 21 & 81 & 90 & 20 & 74 & 6 & 8 & 6 \\
\hline 11 & 1 & 0 & 0 & 47 & 10 & 37 & 4 & 1 & 1 & 203 & 85 & 126 \\
\hline 12 & 1374 & 529 & 1595 & 59 & 3 & 62 & 60 & 15 & 61 & 2081 & 174 & 1130 \\
\hline 13 & 0 & 1 & 3 & 3 & 1 & 1 & 614 & 214 & 342 & 31 & 0 & 4 \\
\hline 14 & 143 & 20 & 203 & 6 & 0 & 5 & 0 & 0 & 4 & 0 & 0 & 0 \\
\hline 15 & 345 & 16 & 154 & 2 & 1 & 4 & 168 & 118 & 144 & 0 & 0 & 0 \\
\hline 16 & 0 & 0 & 0 & 92 & 101 & 177 & 32 & 1 & 7 & 268 & 323 & 1313 \\
\hline 17 & 434 & 21 & 750 & & & & 692 & 93 & 688 & & & \\
\hline 18 & 5 & 13 & 0 & & & & 8499 & 3115 & 11178 & & & \\
\hline 19 & 0 & 1 & 0 & & & & 0 & 0 & 0 & & & \\
\hline 20 & 192 & 22 & 362 & & & & 209 & 59 & 1140 & & & \\
\hline 21 & 11 & 90 & 11 & & & & 1 & 22 & 0 & & & \\
\hline DP & 447,2 & 117,0 & 493,8 & 107,5 & 34,1 & 803,4 & 1931,8 & 674,0 & 2420,6 & 1131,8 & 106,7 & 803,4 \\
\hline
\end{tabular}

Teste de Wilcoxon: valor de Z entre os períodos, no mesmo grupo NS; Mann-Whitney: valor de U, p ara comparar os dois grupos em cada período, NS

Os grupos RTU e controle apresentaram alta prevalência de extrassístoles supraventriculares e ventriculares nos períodos estudados quando foram avaliados o nú-

Revista Brasileira de Anestesiologia

Vol. 54, № 2, Março - Abril, 2004 mero de pacientes que apresentaram ESSV e ESV e a média por hora, sem significância entre eles (Tabelas VI e VII). 
MANDIM, ACHÁ, FONSECA E COL

Tabela VI - Extrassístoles Supraventriculares (ESSV)

\begin{tabular}{|c|c|c|c|c|c|c|}
\hline & \multicolumn{2}{|c|}{ Pré-Operatório (12 h) } & \multicolumn{2}{|c|}{ Intra-Operatório (3 h) } & \multicolumn{2}{|c|}{ Pós-Operatório (12 h) } \\
\hline & RTU & Controle & RTU & Controle & RTU & Controle \\
\hline \multicolumn{7}{|l|}{ Total } \\
\hline $\mathrm{n}(\%)$ & $18(85,7 \%)$ & $15(93,7 \%)$ & $18(85,7 \%)$ & $13(81,2 \%)$ & $16(76,2 \%)$ & $16(100 \%)$ \\
\hline Média/h \pm DP & $19,2 \pm 37,3$ & $4,9 \pm 8,9$ & $17,1 \pm 39,0$ & $7,2 \pm 11,4$ & $21,7 \pm 41,15$ & $3,8 \pm 4,5$ \\
\hline \multicolumn{7}{|l|}{ Isoladas } \\
\hline$n(\%)$ & $18(85,7 \%)$ & $15(93,7 \%)$ & $18(85,7 \%)$ & $13(81,2 \%)$ & $16(76,2 \%)$ & $16(100 \%)$ \\
\hline Média/h $\pm \mathrm{DP}$ & $15,5 \pm 33,5$ & $3,9 \pm 8,1$ & $15,3 \pm 37,3$ & $5,4 \pm 9,02$ & $14,1 \pm 28,9$ & $3,06 \pm 3,33$ \\
\hline \multicolumn{7}{|l|}{ Acopladas } \\
\hline $\mathrm{n}(\%)$ & $9(42,9 \%)$ & $10(62,5 \%)$ & $15(71,4 \%)$ & $11(68,7 \%)$ & $8(38,1 \%)$ & $8(50 \%)$ \\
\hline Média/h \pm DP & $0,75 \pm 1,9$ & $0,23 \pm 0,3$ & $0,76 \pm 1,7$ & $0,52 \pm 1,06$ & $2,37 \pm 9,1$ & $0,15 \pm 0,25$ \\
\hline \multicolumn{7}{|c|}{ Taquicardia supraventricular } \\
\hline$n(\%)$ & $9(42,9 \%)$ & $10(62,5 \%)$ & $3(14,3 \%)$ & $2(12,5 \%)$ & $6(28,6 \%)$ & $5(31,2 \%)$ \\
\hline Média/h \pm DP & $0,14 \pm 0,2$ & $0,11 \pm 0,1$ & $0,05 \pm 0,12$ & $0,16 \pm 0,47$ & $0,07 \pm 0,18$ & $0,08 \pm 0,2$ \\
\hline
\end{tabular}

Teste $t$ de Student $\mathrm{p}>0,05$

Tabela VII - Extrassístoles Ventriculares (ESV)

\begin{tabular}{|c|c|c|c|c|c|c|}
\hline & \multicolumn{2}{|c|}{ Pré-Operatório (12h) } & \multicolumn{2}{|c|}{ Intra-Operatório (3h) } & \multicolumn{2}{|c|}{ Pós-Operatório (12h) } \\
\hline & RTU & Controle & RTU & Controle & RTU & Controle \\
\hline \multicolumn{7}{|l|}{ Total } \\
\hline $\mathrm{n}(\%)$ & $16(76,2 \%)$ & $13(81,2 \%)$ & $17(80,9 \%)$ & $11(68,7 \%)$ & $17(80,9 \%)$ & $13(81,2 \%)$ \\
\hline Média/h \pm DP & $60,3 \pm 160,9$ & $43,1 \pm 94,3$ & $67,3 \pm 224,7$ & $19,8 \pm 35,5$ & $63,4 \pm 201,7$ & $36,2 \pm 66,9$ \\
\hline \multicolumn{7}{|l|}{ Isoladas } \\
\hline $\mathrm{n}(\%)$ & $16(76,2 \%)$ & $12(75 \%)$ & $17(80,9 \%)$ & $11(68,7 \%)$ & $17(80,9 \%)$ & $13(81,2 \%)$ \\
\hline Média/h \pm DP & $59,0 \pm 158,2$ & $42,2 \pm 93,2$ & $58,2 \pm 211,9$ & $19,3 \pm 34,7$ & $52,9 \pm 157,8$ & $35,8 \pm 66,5$ \\
\hline \multicolumn{7}{|l|}{ Acopladas } \\
\hline $\mathrm{n}(\%)$ & $8(38,1 \%)$ & $5(31,2 \%)$ & $8(38,1 \%)$ & $5(31,2 \%)$ & $6(28,6 \%)$ & $5(31,2 \%)$ \\
\hline Média/h \pm DP & $0,62 \pm 1,5$ & $0,41 \pm 1,3$ & $7,8 \pm 27,2$ & $0,35 \pm 1,51$ & $5,14 \pm 22,1$ & $0,22 \pm 0,55$ \\
\hline \multicolumn{7}{|c|}{ Taquicardia ventricular } \\
\hline $\mathrm{n}(\%)$ & $4(19 \%)$ & $3(18,7 \%)$ & $2(9,5 \%)$ & $0(0 \%)$ & $5(23,8 \%)$ & $1(6,25 \%)$ \\
\hline Média/h \pm DP & $0,02 \pm 0,05$ & $0,03 \pm 0,06$ & $0,14 \pm 0,58$ & $0,0 \pm 0,00$ & $0,06 \pm 0,14$ & $0,005 \pm 0,02$ \\
\hline
\end{tabular}

Teste $t$ de Student $p>0,05$

\section{DISCUSSÃO}

No idoso, a perda de células marcapasso e condutoras, por isquemia ou degeneração de estruturas do sistema de condução, levam a distúrbios de condução, como os bloqueios atrioventriculares e bloqueios de ramo e a disritmias cardíacas diversas, sendo que a incidência de alterações eletrocardiográficas aumenta paralelamente com a faixa etária. As disritmias mais freqüentes são: extrassístoles supraventriculares e ventriculares (bloqueios de ramo direito, bloqueio da divisão ântero-superior do ramo esquerdo e bloqueio de ramo esquerdo), alterações da onda Te do segmento $S T^{14}$.
A monitorização contínua possibilita o estudo de todos os tipos de desordens do ritmo cardíaco, sendo relatada incidência acima de $80 \%$, atingindo $100 \%$ quando se realizam cirurgias intra-abdominais, torácicas, cardiovasculares, neurológicas ou de grande porte. Entretanto, quando se realiza apenas inspeção casual, são notadas apenas eventuais disritmias e a incidência é menor que $20 \%{ }^{23}$. Alterações hidroeletrolíticas, metabólicas e autonômicas estão entre as maiores causas ou fatores agravantes das disritmias perioperatórias ${ }^{24}$.

No presente trabalho, observou-se bradicardia durante todos os períodos estudados. A presença de distúrbios da con- 
dução e disritmias nos idosos não significa necessariamente a presença de doença cardíaca ${ }^{16}$. As principais alterações que ocorrem no coração dos pacientes idosos são a hipertrofia ventricular e o aumento do átrio esquerdo, com conseqüências sobre o comportamento do ritmo ${ }^{25}$. O nó sinoatrial sofre redução do volume total, há destruição irregular das áreas periféricas, com substituição por tecido adiposo. A constituição do nó modifica-se com diminuição das células sinoatriais, sem modificação do volume de tecido conjuntivo. Esse comportamento anatômico seria o responsável pela redução da freqüência cardíaca intrínseca ${ }^{26}$

No grupo RTU, um paciente apresentou um número extremamente elevado de pausas sinusais (107 pausas) no intra-operatório. Estas podem ter sido devidas à predominância vagal (doença do nó sinusal) que ocorre quando há bloqueio do nervo simpático pela raquianestesia. O paciente foi tratado com droga parassimpatolítica, porém sem resposta adequada e teve indicação de implante de marcapasso.

Todas as formas de disritmias supraventriculares são encontradas nos idosos e aparentemente a prevalência aumenta com a idade. Dependendo do método empregado para a sua detecção, temos prevalências diferentes. O trabalho de Tammaro e col. ${ }^{27}$ (1983), utilizando eletrocardiografia convencional em 605 pacientes com mais de 60 anos de idade, encontrou disritmias supraventriculares em $33,2 \%$ nos pacientes com mais de 75 anos e em $23,9 \%$ dos pacientes com menos de 75 anos. Conclusões semelhantes foram encontradas em outro trabalho ${ }^{28}$, que estudou pacientes com idades entre 40 e 90 anos e encontrou ESSV em $21,4 \%$ dos pacientes com menos de 60 anos e $74,2 \%$ nos pacientes com mais de 60 anos de idade. Em 32 pacientes com mais de 100 anos de idade, observou-se $31 \%$ de ESSV comparados com prevalência de $4 \%$ na população com idades entre 63 e 95 anos, e média de 75 anos ${ }^{29}$.

Os pacientes do grupo RTU apresentaram uma prevalência de ESSV de $85,7 \%$ no pré e intra-operatório e $76,2 \%$ no pós-operatório. No grupo controle, houve uma prevalência de $93,7 \%$ no pré-operatório, $81,2 \%$ no intra-operatório e $100 \%$ no pós-operatório (Tabela VI). As ESSV não foram responsáveis por maior incidência de morbidade, o que está de acordo com outros autores ${ }^{28,29}$.

Nenhum paciente apresentou fibrilação atrial, que é a disritmia mais comum nos idosos e está presente em $0,4 \%$ da população geral e em 3\% a 5\% daqueles com mais de 65 anos ${ }^{30}$.

As ESV ocorrem em $30 \%$ a $84 \%$ dos indivíduos com coração aparentemente normal à avaliação pelos exames clínicos e não invasivos (eletrocardiograma, teste de esforço e ecocardiograma) ${ }^{31}$. No estudo de Rasmussen e col. ${ }^{32}$ (1985), a prevalência das ESV aumentou com a idade, sendo de $31 \%$ no grupo de 20 a 39 anos, $68 \%$ no grupo de 40 a 59 anos e de $84 \%$ nos grupo de 60 a 79 anos. Amaioria ( $80 \%$ ) apresentava menos de uma extrassístole ventricular/hora. As extrassístoles ventriculares eram geralmente raras, monomórficas e isoladas nesses pacientes.

Dietz e col. ${ }^{33}$ (1987) relataram que $87,2 \%$ dos idosos com média de idade de 80 anos tinham extrassístoles freqüentes, quando estudados pelo Sistema Holter. Wajngarten e col. ${ }^{25}$,
(1990) estudaram 26 pacientes com mais de 70 anos de idade, $77 \%$ tinham ESV, freqüentes em $23 \%$ sendo polimórficas em $50 \%$. Taquicardia ventricular não sustentada ocorreu em $11,5 \%$ dos pacientes. Apesar de observar-se, ao ecocardiograma, um aumento da massa ventricular com a idade, não houve correlação com o aparecimento de disritmias ventriculares.

Gravações de ECG contínuo foram usadas em dois estudos de disritmias em um total de 254 pacientes submetidos à cirurgia não cardíaca ${ }^{15,34}$. A incidência de disritmias foi de $70 \%$, sendo $28 \%$ ventriculares. O primeiro estudo ${ }^{34}$ foi conduzido em 154 pacientes submetidos à cirurgia não cardíaca que revelaram uma incidência de disritmias de $62 \%$. Esta incidência foi maior durante anestesia geral versus anestesia regional ( $66 \%$ vs. $52 \%$ ), cirurgia neurológica e torácica versus cirurgia periférica ( $100 \%, 90 \%$ vs. $56 \%)$, e pacientes intubados versus não intubados ( $72 \%$ vs. $44 \%$ ). Vinte e um porcento das disritmias foram ventriculares (extrassístoles em $18 \%$ e taquicardia ventricular $3 \%$ ). Surpreendentemente, doença cardíaca preexistente não apresentou influência na incidência de disritmias ( $62 \%$ vs. $59 \%$ ). O outro estudo ${ }^{15}$ encontrou uma incidência de $84 \%$ de disritmias em 100 pacientes submetidos à cirurgia não cardíaca. A incidência intra-operatória foi significantemente maior do que a fase de pré-indução ( $84 \%$ vs. $28 \%$ ), particularmente durante a intubação e extubação traqueal, quando ocorreram $72 \%$ das disritmias, uma incidência de $43 \%$ de disritmias ventriculares, maior em pacientes com doença cardíaca preexistente (60\% vs. $37 \%$ ). Esses estudos, no entanto, não identificaram as faixas etárias.

Disritmias ventriculares isoladas, em pacientes assintomáticos e com função ventricular normal, não foram fatores de risco para complicações cardiovasculares perioperatórias. Entretanto, disritmias ventriculares complexas, freqüentes e com função ventricular anormal foram observadas no período perioperatório, principalmente em pacientes com antecedentes de cardiopatias e levaram a graves complicações e alta mortalidade cardíaca. Entretanto, pacientes com antecedentes de cardiopatia, com função ventricular anormal e presença de disritmias ventriculares freqüentes e complexas tiveram maior número de complicações e com uma elevada taxa de mortalidade ${ }^{35}$. No presente trabalho verificou-se que as ESV estiveram presentes, no grupo RTU versus controle em: ( $76,2 \%$ vs. $81,2 \%)$ no pré-operatório, $(80,9 \%$ vs. $68,7 \%)$ no intra e pós-operatório $(80,9 \%$ vs. $81,2 \%)$.

Diferentemente do trabalho realizado por Marshall e col. ${ }^{36}$ (1972), foi verificado em nosso estudo que as disritmias ventriculares (isoladas, pareadas e taquicardia ventricular) não foram responsáveis pelo aparecimento de complicações cardiovasculares, tanto no intra quanto no pós-operatório e que apesar da alta prevalência de disritmias supraventriculares e ventriculares nesta faixa etária, não houve diferença significativa quando foi realizada a raquianestesia.

A cirurgia de RTU da próstata não apresentou aumento do número de disritmias em comparação ao grupo controle e nenhum paciente apresentou sinais de síndrome da RTU durante o estudo. 
As disritmias cardíacas após cirurgias extracardíacas são freqüentes e podem ser precipitadas por outras complicações não inerentes ao coração, como: sangramentos, infecções, desequilíbrios ácido-básicos, hidroeletrolíticos e hipoxemia ${ }^{36}$. A realização da eletrocardiografia dinâmica (Holter) antes da cirurgia pode estar indicada frente à suspeita de isquemia miocárdica silenciosa em paciente com doença coronariana. Diversos trabalhos demonstraram a relação entre o infradesnivelamento do segmento ST no pré-operatório e a freqüência de eventos isquêmicos. Mangano e col. ${ }^{35} 1990$, demonstraram incidência de $25 \%$ de episódios isquêmicos no período intra-operatório, em 200 pacientes estudados. Em estudo envolvendo 474 homens com alto risco ou sabidamente portadores de doença arterial coronariana, utilizando monitorização com Holter de dois canais em cirurgia eletiva não cardíaca, dois dias antes da cirurgia, durante e dois dias após, observou que a isquemia miocárdica registrada pelo Holter antes da cirurgia aumentou em 9,2 vezes o risco de evento isquêmico pós-operatório ${ }^{35}$.

O interesse na monitorização perioperatória da isquemia miocárdica reflete a possibilidade de usá-la como um fator de previsão de resultados cardíacos adversos, tais como o infarto agudo do miocárdio, a falência cardíaca ou morte. Um número de fatores de risco tem sido identificado em populações selecionadas ${ }^{37,38}$. No entanto, graves resultados adversos (morte, infarto ou angina) são raros, mesmo em pacientes de alto risco, mas a detecção de isquemia miocárdica silenciosa pode ajudar a identificar essa subpopulação com risco aumentado de efeitos cardiovasculares adversos.

Evans e col. ${ }^{39}$ (1991) quantificaram as alterações hemodinâmicas durante RTU usando transdutor de Doppler esofágico e verificaram que ocorreu um aumento na pós-carga do ventrículo esquerdo indicando um aumento do trabalho miocárdico e da demanda de oxigênio que pode resultar em isquemia miocárdica, contribuindo para o aumento na morbidade e mortalidade cardiovascular. A freqüência cardíaca e volume sistólico apresentaram diminuição progressiva nos primeiros 30 minutos de cirurgia, resultando em redução no débito cardíaco, com um aumento significante na pós-carga do VE desde o início do procedimento. Estes achados demonstraram que as respostas hemodinâmicas que não são detectáveis usando métodos convencionais de monitorização ocorrem durante a RTU. Aumento da pós-carga do VE indica aumento no trabalho miocárdico e na demanda de $\mathrm{O}_{2}$ o que pode resultar em isquemia miocárdica, contribuindo para o aumento na morbidade e mortalidade cardiovascular que tem sido encontrada após RTU.

No estudo de Dobson e col. ${ }^{40}$ (1994) foram analisadas as variáveis hemodinâmicas dos pacientes submetidos a RTU sob anestesias geral e espinhal e concluíram que ambas as técnicas anestésicas estão associadas com grandes alterações nas variáveis hemodinâmicas, logo após a indução da anestesia, sendo que estas são maiores com a anestesia geral. Também observaram que o período de ressecção da próstata não está associado com alterações hemodinâmicas significantes.

Edwards e col. ${ }^{41}$ (1995) estudaram a incidência e duração da isquemia miocárdica perioperatória usando ECG ambulatorial em 100 pacientes submetidos à cirurgia transuretral da próstata, aleatórios para receber anestesia geral ou espinhal. A incidência total de isquemia miocárdica passou de $18 \%$ no pré-operatório para $26 \%$ no pós-operatório. Pacientes com doença cardíaca isquêmica tiveram uma incidência significantemente maior de isquemia miocárdica após a operação do que os pacientes sem doença isquêmica previamente conhecida. Houve um aumento na incidência e duração da isquemia miocárdica após a operação com ambas as técnicas anestésicas; no entanto, não houve diferença significativa entre as duas.

Em estudo prospectivo e aleatório, Shalev e col. ${ }^{42}$ (1999) compararam a morbidade e a incidência de infarto agudo do miocárdio em pacientes submetidos a RTU de próstata, prostatectomia convencional e outras cirurgias não cardiacas, $A$ incidência de IAM após prostatectomia, com ambas as técnicas, foi maior que $6 \%$, comparadas com as demais cirurgias, que é de 2,5\%. Não houve diferença estatística na mortalidade que foi de $14,4 \%$ na RTU e $8,5 \%$ na prostatectomia convencional.

No presente estudo, as incidências de alterações do segmento ST para os grupos RTU versus controle foram, no pré-operatório ( $19 \%$ vs $18,7 \%$ ), no intra-operatório (4,8\% vs $18,7 \%$ ) e no pós-operatório ( $14,3 \%$ vs. $18,7 \%)$, sem diferença estatística entre os grupos. Estes resultados são comparáveis aos encontrados no estudo realizado por Edwards e col. ${ }^{41}$ (1995).

As disritmias supraventriculares, ventriculares e alterações do segmento ST não foram responsáveis por complicações no intra e no pós-operatório tanto no grupo RTU como no grupo controle. Acreditamos que a presença de um elevado número de extrassístoles não contra-indica uma cirurgia de ressecção transuretral da próstata em pacientes idosos.

Concluímos que os paciente idosos apresentam alta prevalência de disritmias supraventriculares e ventriculares e que o número total de disritmias supraventriculares e ventriculares e alterações do segmento ST presentes no período pré-operatório não foi alterado pela cirurgia de ressecção transuretral da próstata, bem como herniorrafia inguinal, nos períodos intra e pós-operatório. 


\section{Cardiac Arrhythmias and ST Changes in the Perioperative Period of Elderly Patients Submitted to Transurethral Prostatectomy Under Spinal Anesthesia. Comparative Study}

Beatriz Lemos da Silva Mandim, TSA, M.D.; Renato Enrique Sologuren Achá, M.D.; Neuber Martins Fonseca, TSA, M.D.; Fabiano Zumpano, M.D

\section{INTRODUCTION}

There is no clear definition for "old", "elderly" or "old age", as there is no specific clinic marker for geriatric patients since aging is not an abrupt, but rather a continuous process ${ }^{1}$. Geriatric population is uniquely heterogeneous ${ }^{2}$. The World Health Organization, based on socio-economic factors, considers elderly all individuals aged 65 years or above. In Brazil, according to the law (8842, January 1994) elderly is all individuals aged 60 years or above ${ }^{3}$. In general, physiological functions decrease $1 \%$ a year after 30 years of age, and at 70 , baseline metabolism is $40 \%$ of normal ${ }^{4}$.

Common preoperative elderly diseases are ECG changes, cardiovascular arteriosclerosis, previous myocardial infarction, congestive heart failure, liver and kidney diseases ${ }^{5}$. Elderly represent $25 \%$ of surgical patients and it is estimated that $50 \%$ of people above 65 years of age will undergo one surgery before death ${ }^{6}$.

Several coronary disease patients have normal preoperative $\mathrm{ECG}^{7}$. Silent myocardial ischemia (SMI) detected by long duration dynamic ECG (Holter System) is common during daily activities of asymptomatic patients with coronary disease and positive treadmill stress tests ${ }^{8}$. In angina patients, $80 \%$ of ischemia are silent episodes ${ }^{9}$. There is a high incidence of SMI in the first postoperative week, varying $21 \%{ }^{10}$ to $60 \%{ }^{11}$. The first clinical sign of perioperative ischemia is low cardiac output ${ }^{11,12}$, arterial hypotension or arrhythmia ${ }^{13}$.

The incidence of ECG changes in elderly increases with age, being observed: T wave and ST changes, supraventricular (SVPC) and ventricular (VCP) premature complexes and atrial fibrillation, intraventricular conduction abnormalities (right bundle branch block, left bundle branch block and first-degree atrioventricular block. Some are frequent in elderly patients (premature complexes, atrial fibrillation, left bundle branch block, intraventricular conduction abnormalities and ST changes), while others are better correlated to the presence of associated anatomic changes (pathological $Q$ wave, right bundle branch block and left bundle branch block) ${ }^{14}$. Bertrand et al. ${ }^{15}(1971)$ have reported $48 \%$ incidence of arrhythmias during general anesthesia recovery, being $28 \%$ ventricular arrhythmias.

As people age, there are physiological heart changes affecting myocardium and conducting system, among others. These changes are not dependent on pathological changes, which are frequent in elderly. Several age-related heart electrophysiological changes are similar to those caused by diseases ${ }^{16}$. Structural changes lead to collagen tissue and myocardial amyloidal matter increase and specific conduction tissue cells ${ }^{17}$ and adrenergic stimulation sensitivity decrease $^{18}$

Transurethral prostatectomy (TUP) is performed in elderly patients and is associated to considerable morbidity. Post-TUP cardiovascular deaths are $0.5 \%$ to $1 \%$, increasing to more than $2 \%$ in patients above 80 years of age ${ }^{19,20}$. There are studies evaluating ST changes or acute myocardial infarction (AMI) during transurethral prostatectomy, but no studies were found evaluating the prevalence of intraoperative arrhythmias using dynamic ECG (Holter System) in patients submitted to this surgery under spinal anesthesia.

This study aimed at evaluating the prevalence of perioperative arrhythmias and ST changes through the Holter System in elderly patients submitted to transurethral prostatectomy (TUP) and inguinal hernia repair under spinal anesthesia.

\section{METHODS}

After the Universidade Federal, Uberlândia Ethics Committee approval, participated in this randomized study 21 patients with benign prostate hypertrophy aged 65 to 84 years and submitted to TUP (Group TUP), and 16 patients with unilateral inguinal hernia (control group) aged 65 to 86 years and submitted to inguinal hernia repair under spinal anesthesia, in the period November 2001 through August 2002. All patients were informed about the research objectives and have signed the informed consent. Exclusion criteria were positive serology for Chagas disease, ST changes at ECG, liver and kidney diseases, anemia, infections and spinal anesthesia counterindications.

Patients were preoperatively evaluated by history, physical, lab and 12-lead ECG evaluation. Myocardial ischemia was defined as ST horizontalization or depression for $1 \mathrm{~mm}$ or more or ST increase of $2 \mathrm{~mm}$ or more during 60 seconds or more, followed by return to baseline for at least 1 minute ${ }^{21}$.

Long duration dynamic ECG (Holter System) with Dynamics 3000 recorder was analyzed by the Cardiosystem program, release ALT V5 08C, 12 hours before anesthesia; during the surgical procedure from anesthesia beginning to completion with 3 hours duration, based on bupivacaine's duration which is 180 minutes $^{22}$; and 12 hours after anesthesia.

Cardiac rhythm: heart rate (bradycardia and sinus tachycardia); presence, classification (supraventricular or ventricular) and complexity (isolated, paired or cluster premature complexes) of arrhythmias, presence of atrioventricular blocks and/or of His bundle branches and ST changes were evaluated, considering pre, intra and postoperative periods. Patients of both groups were not premedicated. Usual anti-hypertensive and cardiovascular drugs doses, except for aspirin, were maintained. Venous hydration was installed in the operating room 30 minutes before anesthesia with 500 $\mathrm{mL}$ lactated Ringer's. Monitoring consisted of cardioscope at 
CM5 lead, sphygmomanometer to control blood pressure by the Riva-Rocci method at 5-minute intervals, and pulse oximetry for continuous oxygen saturation $\left(\mathrm{SpO}_{2}\right)$ reading. Spinal anesthesia was performed with patients in the right lateral position and previous skin infiltration with $1 \%$ lidocaine at puncture site. The needle was introduced medially at $L_{2}-L_{3}$ or $L_{3}-L_{4}$ with cephalad bevel, a $25 \mathrm{G}$ Quincke needle was used. After spinal space identification by the presence of CSF, $14 \mathrm{mg}$ of $0.5 \%$ hyperbaric bupivacaine were injected.

Immediately after injection, patients were placed in the supine position until blockade is established evaluated by the loss of sensitivity to cold test. When the block reached $T_{10}$ level patients were placed in the surgical position. If blood pressure reached $20 \%$ below baseline, ephedrine $(10 \mathrm{mg}$ ) was administered. Postoperative analgesia was systematically induced with $1 \mathrm{~g}$ intravenous dipirone every 6 hours. TUP was performed in the lythotomy position with continuous room temperature distilled water irrigation, up to $50 \mathrm{~cm}$ higher with regard to patients.

Non-parametric Wilcoxon's test was used for related samples to compare each group in the pre, intra and postoperative periods. Mann-Whitney's $U$ test was used for independent samples to compare both groups in each evaluated period. Student's $t$ test was used for independent samples when age, prevalence of bradycardia, arrhythmias and ST changes, and arrhythmias complexity were compared between groups.

For all tests, 0.05 or $5 \%(p<0.05)$ was considered null hypothesis rejection level and significant values are identified with a asterisk $\left({ }^{*}\right)$.

\section{RESULTS}

Demographics data and physical status were homogeneous between groups (Table I).

During preoperative evaluation it has been observed that 2 group TUP patients had already previous acute myocardial infarction as compared to none in control group, with statistical significance. Other parameters were similar between groups and without statistical significance (Table II).
Table I - Demographics Data

\begin{tabular}{lcc}
\hline Parameters & $\begin{array}{c}\text { Group TUP } \\
(\mathrm{n}=21)\end{array}$ & $\begin{array}{c}\text { Group Control } \\
(\mathrm{n}=16)\end{array}$ \\
\hline Age (years) * & $73.05 \pm 6.79$ & $70.5 \pm 6.11$ \\
Weight $(\mathrm{kg})^{*}$ & $69.24 \pm 13.13$ & $65.13 \pm 10.29$ \\
Height $(\mathrm{m}){ }^{*}$ & $1.69 \pm 0.04$ & $1.67 \pm 0.07$ \\
Physical Status & & \\
$\quad$ ASA II & $16(76.19 \%)$ & $14(87.50 \%)$ \\
$\quad$ ASA III & $5(23.81 \%)$ & $2(12.50 \%)$ \\
\hline
\end{tabular}

* Data expressed in Mean \pm SD. Student's $t$ test: $p>0.05$

Table II - Preoperative Clinical Evaluation

\begin{tabular}{lcc}
\hline Parameters & $\begin{array}{c}\text { Group TUP } \\
(\mathrm{n}=21)\end{array}$ & $\begin{array}{c}\text { Group Control } \\
(\mathrm{n}=16)\end{array}$ \\
\hline Acute myocardial infarction & $2(9.52 \%)^{*}$ & $0(0 \%)$ \\
Systemic arterial hypertension & $9(42.86 \%)$ & $8(50 \%)$ \\
Diabetes mellitus & $1(4.76 \%)$ & $2(12.50 \%)$ \\
Smoking & $3(14.28 \%)$ & $2(12.50 \%)$ \\
\hline
\end{tabular}

* Student's $t$ test: $p<0.05$

Preoperative evaluations were homogeneous between groups (Table III).

Table III - Preoperative Evaluation

\begin{tabular}{lcc}
\hline Parameters & $\begin{array}{c}\text { Group TUP } \\
(\mathrm{n}=21)\end{array}$ & $\begin{array}{c}\text { Group Control } \\
(\mathrm{n}=16)\end{array}$ \\
\hline Hemoglobin * $^{*}$ & $13.9 \pm 1.6$ & $15.2 \pm 1.7$ \\
Glycemia * $^{*}$ & $111.7 \pm 36.1$ & $115.3 \pm 27.5$ \\
Normal ECG & $2(9.5 \%)$ & $4(25.0 \%)$ \\
ECG with arrhythmias & $12(57.1 \%)$ & $6(37.5 \%)$ \\
ECG indirect signs of acute & $14(66.7 \%)$ & $7(43.7 \%)$ \\
myocardial infarction & & \\
\hline
\end{tabular}

* Data expressed in Mean \pm SD. Student's $t$ test: $p>0.05$

Table IV shows the incidence of sinus bradycardia $(H R<60)$, sinus pauses and ST changes, comparing TUP and control groups in the pre, intra and postoperative periods, as well as

Table IV - Sinus Bradycardia, Sinus Pauses and ST Changes in the Pre, Intra and Postoperative Periods for Both Groups

\begin{tabular}{|c|c|c|c|c|c|c|}
\hline & \multicolumn{2}{|c|}{ Preoperative (12 h) } & \multicolumn{2}{|c|}{ Intraoperative (3 h) } & \multicolumn{2}{|c|}{ Postoperative (12 h) } \\
\hline & $\begin{array}{c}\text { TUP } \\
(n=21)\end{array}$ & $\begin{array}{l}\text { Control } \\
(n=16)\end{array}$ & $\begin{array}{c}\text { TUP } \\
(n=21)\end{array}$ & $\begin{array}{l}\text { Control } \\
(n=16)\end{array}$ & $\begin{array}{c}\text { TUP } \\
(n=21)\end{array}$ & $\begin{array}{c}\text { Control } \\
(n=16)\end{array}$ \\
\hline \multicolumn{7}{|l|}{ Sinus bradycardia } \\
\hline $\mathrm{HR}<60-\mathrm{n}(\%)$ & $18(87 \%)$ & $12(75 \%)$ & $20(95 \%)$ & $11(68.7 \%)$ & $18(86 \%)$ & $11(68.7 \%)$ \\
\hline Mean $/ \mathrm{h} \pm \mathrm{SD}$ & $46 \pm 10.4$ & $52.6 \pm 10.1$ & $43.7 \pm 10.2$ & $52.7 \pm 12.5$ & $46.7 \pm 10.8$ & $54.6 \pm 13.1$ \\
\hline \multicolumn{7}{|l|}{ Sinus pauses } \\
\hline Pauses - n (\%) & $2(9 \%)$ & $1(6.2 \%)$ & $1(5 \%)$ & $1(6.2 \%)$ & $1(5 \%)$ & $2(12.5 \%)$ \\
\hline Mean $/ \mathrm{h} \pm \mathrm{SD}$ & $0.003 \pm 0.02$ & $0.03 \pm 0.12$ & $1.7 \pm 7.8^{*}$ & $0.06 \pm 0.25$ & $0.007 \pm 0.04$ & $0.01 \pm 0.03$ \\
\hline \multicolumn{7}{|l|}{ ST changes } \\
\hline $\mathrm{ST}-\mathrm{n}(\%)$ & $4(19 \%)$ & $3(18.7 \%)$ & $1(4.8 \%)$ & $3(18.7 \%)$ & $3(14.3 \%)$ & $3(18.7 \%)$ \\
\hline Mean $/ \mathrm{h} \pm \mathrm{SD}$ & $0.04 \pm 0.11$ & $0.15 \pm 0.34$ & $0.02 \pm 0.07$ & $0.12 \pm 0.3$ & $0.03 \pm 0.08$ & $0.12 \pm 0.25$ \\
\hline
\end{tabular}

* Student's $t$ test $p<0.05$ 
means by studied hour in each period, without statistical differences between groups, except for mean intraoperative sinus pauses in group TUP, where 1 patient had 107 pauses.

Table V shows total number of supraventricular and ventricular premature complexes in both groups and no statistical dif- ferences were found when periods of each group and both groups in each period were compared.

There has been a high incidence of supraventricular and ventricular premature complexes in both groups during the studied period, without statistical differences (Tables VI and VII).

Table V - Total Number of Supraventricular (SVPC) and Ventricular (VPC) Premature Complex in Both Groups

\begin{tabular}{|c|c|c|c|c|c|c|c|c|c|c|c|c|}
\hline & \multicolumn{6}{|c|}{ Supraventricular Premature Complex } & \multicolumn{6}{|c|}{ Ventricular Premature Complex } \\
\hline & \multicolumn{3}{|c|}{ Group TUP } & \multicolumn{3}{|c|}{ Group Control } & \multicolumn{3}{|c|}{ Group TUP } & \multicolumn{3}{|c|}{ Group Control } \\
\hline & Pre (12h) & Intra (3h) & Post (12h) & Pre (12h) & Intra (3h) & Post (12h) & Pre (12h) & Intra (3h) & Post (12h) & Pre (12h) & Intra (3h) & Post (12h) \\
\hline 1 & 63 & 7 & 14 & 54 & 98 & 49 & 0 & 0 & 3 & 2 & 2 & 1 \\
\hline 2 & 2 & 1 & 7 & 20 & 0 & 15 & 29 & 6 & 41 & 5 & 0 & 12 \\
\hline 3 & 94 & 74 & 90 & 0 & 0 & 2 & 0 & 0 & 0 & 110 & 31 & 117 \\
\hline 4 & 1650 & 168 & 1674 & 56 & 17 & 32 & 3211 & 149 & 440 & 161 & 13 & 99 \\
\hline 5 & 4 & 0 & 0 & 8 & 7 & 9 & 0 & 4 & 0 & 1 & 1 & 3 \\
\hline 6 & 335 & 67 & 415 & 445 & 62 & 158 & 1469 & 376 & 1457 & 1173 & 22 & 1344 \\
\hline 7 & 11 & 8 & 23 & 19 & 5 & 66 & 3 & 3 & 6 & 4199 & 290 & 2804 \\
\hline 8 & 128 & 24 & 129 & 34 & 15 & 17 & 94 & 41 & 389 & 30 & 4 & 2 \\
\hline 9 & 31 & 7 & 19 & 90 & 21 & 81 & 90 & 20 & 74 & 6 & 8 & 6 \\
\hline 10 & 24 & 4 & 24 & 4 & 1 & 9 & 34 & 2 & 1 & 0 & 0 & 0 \\
\hline 11 & 1 & 0 & 0 & 47 & 10 & 37 & 4 & 1 & 1 & 203 & 85 & 126 \\
\hline 12 & 1374 & 529 & 1595 & 59 & 3 & 62 & 60 & 15 & 61 & 2081 & 174 & 1130 \\
\hline 13 & 0 & 1 & 3 & 3 & 1 & 1 & 614 & 214 & 342 & 31 & 0 & 4 \\
\hline 14 & 143 & 20 & 203 & 6 & 0 & 5 & 0 & 0 & 4 & 0 & 0 & 0 \\
\hline 15 & 345 & 16 & 154 & 2 & 1 & 4 & 168 & 118 & 144 & 0 & 0 & 0 \\
\hline 16 & 0 & 0 & 0 & 92 & 101 & 177 & 32 & 1 & 7 & 268 & 323 & 1313 \\
\hline 17 & 434 & 21 & 750 & & & & 692 & 93 & 688 & & & \\
\hline 18 & 5 & 13 & 0 & & & & 8499 & 3115 & 11178 & & & \\
\hline 19 & 0 & 1 & 0 & & & & 0 & 0 & 0 & & & \\
\hline 20 & 192 & 22 & 362 & & & & 209 & 59 & 1140 & & & \\
\hline 21 & 11 & 90 & 11 & & & & 1 & 22 & 0 & & & \\
\hline Mean & 230.8 & 51.1 & 260.6 & 58.7 & 21.4 & 45.2 & 724.2 & 201.9 & 760.8 & 516.9 & 59.6 & 435.1 \\
\hline SD & 447.2 & 117.0 & 493.8 & 107.5 & 34.1 & 803.4 & 1931.8 & 674.0 & 2420.6 & 1131.8 & 106.7 & 803.4 \\
\hline
\end{tabular}

Wilcoxon's Test: $Z$ value among periods in the same group NS; Mann-Whitney: $U$ value, to comp are both groups in each period, NS

Table VI - Supraventricular Premature Complex (SVPC)

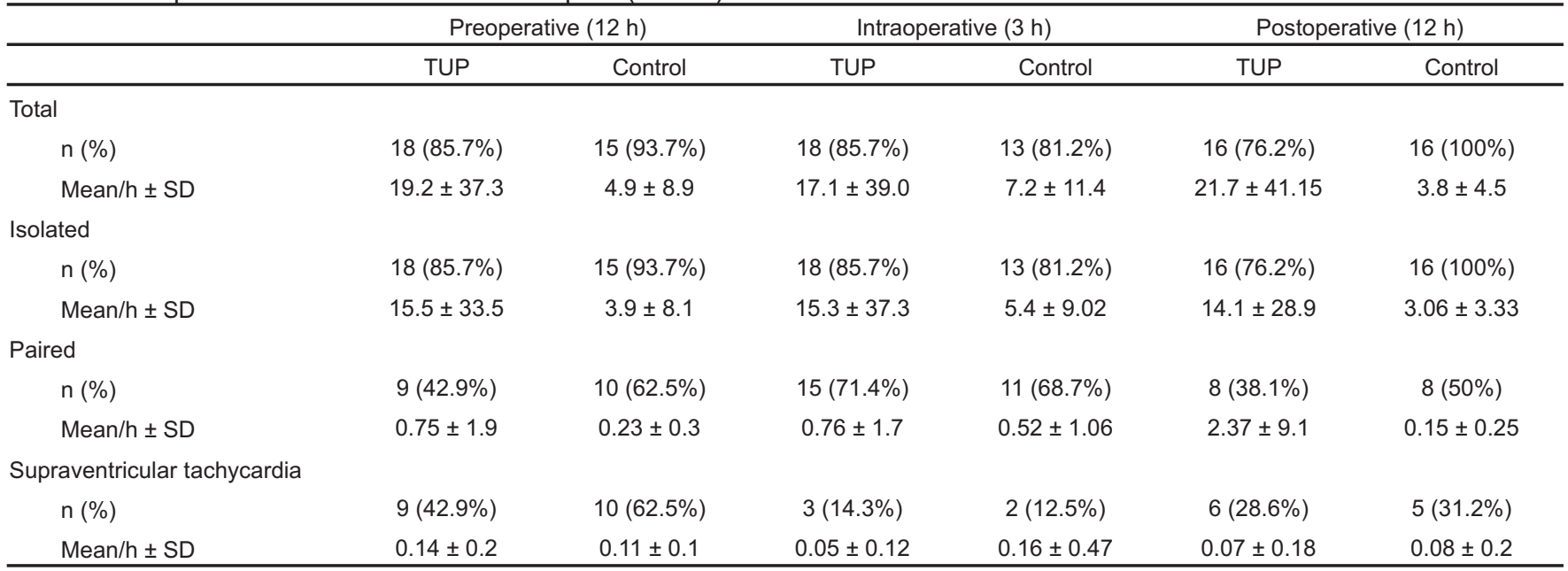

Student's $t$ test $p>0.05$ 
Table VII - Ventricular Premature Complex (VPC)

\begin{tabular}{|c|c|c|c|c|c|c|}
\hline & \multicolumn{2}{|c|}{ Preoperative (12 h) } & \multicolumn{2}{|c|}{ Intraoperative (3 h) } & \multicolumn{2}{|c|}{ Postoperative (12h) } \\
\hline & TUP & Control & TUP & Control & TUP & Control \\
\hline \multicolumn{7}{|l|}{ Total } \\
\hline $\mathrm{n}(\%)$ & $16(76.2 \%)$ & $13(81.2 \%)$ & $17(80.9 \%)$ & $11(68.7 \%)$ & $17(80.9 \%)$ & $13(81.2 \%)$ \\
\hline Mean/h \pm SD & $60.3 \pm 160.9$ & $43.1 \pm 94.3$ & $67.3 \pm 224.7$ & $19.8 \pm 35.5$ & $63.4 \pm 201.7$ & $36.2 \pm 66.9$ \\
\hline \multicolumn{7}{|l|}{ Isolated } \\
\hline $\mathrm{n}(\%)$ & $16(76.2 \%)$ & $12(75 \%)$ & $17(80.9 \%)$ & $11(68.7 \%)$ & $17(80.9 \%)$ & $13(81.2 \%)$ \\
\hline Mean/h \pm SD & $59.0 \pm 158.2$ & $42.2 \pm 93.2$ & $58.2 \pm 211.9$ & $19.3 \pm 34.7$ & $52.9 \pm 157.8$ & $35.8 \pm 66.5$ \\
\hline \multicolumn{7}{|l|}{ Paired } \\
\hline$n(\%)$ & $8(38.1 \%)$ & $5(31.2 \%)$ & $8(38.1 \%)$ & $5(31.2 \%)$ & $6(28.6 \%)$ & $5(31.2 \%)$ \\
\hline Mean/h \pm SD & $0.62 \pm 1.5$ & $0.41 \pm 1.3$ & $7.8 \pm 27.2$ & $0.35 \pm 1.51$ & $5.14 \pm 22.1$ & $0.22 \pm 0.55$ \\
\hline \multicolumn{7}{|c|}{ Supraventricular tachycardia } \\
\hline $\mathrm{n}(\%)$ & $4(19 \%)$ & $3(18.7 \%)$ & $2(9.5 \%)$ & $0(0 \%)$ & $5(23.8 \%)$ & $1(6.25 \%)$ \\
\hline Mean/h \pm SD & $0.02 \pm 0.05$ & $0.03 \pm 0.06$ & $0.14 \pm 0.58$ & $0.0 \pm 0.00$ & $0.06 \pm 0.14$ & $0.005 \pm 0.02$ \\
\hline
\end{tabular}

Student's $t$ test $p>0.05$

\section{DISCUSSION}

The loss of pacemaker and conducting cells in the elderly, by ischemia or conducting system structures degeneration, leads to conduction abnormalities, such as atrioventricular blocks and bundle branch blocks, as well as several arrhythmias; the incidence of ECG changes increases in parallel with increased age. Most frequent arrhythmias are: supraventricular and ventricular premature complexes (right bundle branch block, left blunde branch - partial or complete block), T wave and ST changes ${ }^{14}$.

Continuous monitoring allows for the study of all types of cardiac arrhythmias, being reported an incidence of more than $80 \%$, reaching $100 \%$ when intra-abdominal, chest, cardiovascular, neurological or major surgeries are performed. However, when there is casual inspection, only sporadic arrhythmias are observed and the incidence is below $20 \%{ }^{23}$. Hydroelectrolytic, metabolic and autonomic disturbances are among the main causes or worsening factors for perioperative arrhythmias ${ }^{24}$.

Our study has observed bradycardia during all studied periods. The presence of conducting disorders and arrhythmias in the elderly does not necessarily mean the presence of heart disease ${ }^{16}$. Major elderly heart changes are ventricular hypertrophy and left atrium increase, with consequences on cardiac rhythm ${ }^{25}$. Sinus node has its total volume decreased, there is irregular destruction of peripheral areas with replacement by fatty tissue. Node constitution is changed with sinus cells decrease without connective tissue volume changes. This anatomic change would be responsible for intrinsic heart rate decrease ${ }^{26}$.

One group TUP patient presented a very high number of intraoperative sinus pauses (107 pauses), which might have been due to vagal predominance (sick sinus syndrome) following spinal anesthesia sympathetic block. Patient was treated with parasympatholytic drug however without adequate response and was referred to pacemaker implant. All supraventricular arrhythmias are found in the elderly and it seems that prevalence increases with age. Depending on the method used to detect arrhythmias, there are different prevalences. Tammaro et al. ${ }^{27}$ (1983), using conventional ECG in 605 patients above 60 years of age, have found supraventricular arrhythmias in $33.2 \%$ of patients above 75 years of age and in $23.9 \%$ of patients with less than 75 years of age. Similar conclusions were drawn by a different study ${ }^{28}$ with patients aged 40 to 90 years, which has found SVPC in $21.4 \%$ of patients below 60 years of age and in $74.2 \%$ of patients above 60 years of age. It has been observed $31 \%$ of SVPC in patients above 100 years of age as compared to $4 \%$ in the population aged 63 to 95 years and mean of 75 years of age ${ }^{29}$.

Group TUP patients had $85.7 \%$ pre and intraoperative SVPC and $76.2 \%$ postoperative. Control group patients had $93.7 \%$ preoperative, $81.2 \%$ intraoperative and $100 \%$ postoperative SVPC (Table VI). SVPC were not responsible for a higher incidence of morbidity, which is in line with other authors ${ }^{28,29}$. No patient had atrial fibrillation, which is the most common elderly arrhythmia and is present in $0.4 \%$ of general population and in $3 \%$ to $5 \%$ of people above 65 years of age ${ }^{30}$.

VPC was present in $30 \%$ to $84 \%$ of individuals with seemingly normal hearts at clinical and noninvasive tests (ECG, stress test and echocardiogram) ${ }^{31}$. In a study by Rasmussen et al. ${ }^{32}$ (1985) VPC has increased with age, being $31 \%$ in the group aged 20 to 39 years, $68 \%$ in the group aged 40 to 59 years and $84 \%$ in the group aged 60 to 79 years. Most $(80 \%)$ had less than one ventricular premature complex/hour. Ventricular premature complex was in general uncommon, monomorphic and isolated in those patients.

Dietz et al. ${ }^{33}$ (1987) have reported that $87.2 \%$ of elderly patients with mean age of 80 years had frequent premature

Revista Brasileira de Anestesiologia Vol. 54, N², Março - Abril, 2004 
complex when studied by Holter. Wajngarten et al. ${ }^{25}$ (1990) have studied 26 patients above 70 years of age, where $77 \%$ had VPC in $23 \%$ they were frequent and in $50 \%$ they were polymorphic. Non-sustainable ventricular tachycardia was present in $11.5 \%$ of patients. Although ventricular mass increase with age was seen at echocardiogram, there has been no correlation with ventricular arrhythmias.

Continuous ECG records were used in two arrhythmia studies in a total of 254 patients submitted to non-cardiac surgeries ${ }^{15,34}$. The incidence of arrhythmias was $70 \%$, being $28 \%$ ventricular. The first study ${ }^{34}$ was performed with 154 patients submitted to non-cardiac surgery, who presented a $62 \%$ incidence of arrhythmias. This incidence was higher during general anesthesia versus regional anesthesia ( $66 \%$ vs. $52 \%)$, neurological and chest surgeries versus peripheral surgeries $(100 \%, 90 \%$ vs. $56 \%)$ and intubated versus non-intubated patients ( $72 \%$ vs. $44 \%)$. Ventricular arrhythmias (18\% premature complexes and $3 \%$ ventricular tachycardia) were responsible for $21 \%$ of cases. Surprisingly, preexistent heart disease had no influence in the incidence of arrhythmias (62\% vs. $59 \%$ ). Adifferent study ${ }^{15}$ has found $84 \%$ of arrhythmias in 100 patients submitted to non-cardiac surgeries. Intraoperative incidence was significantly higher as compared to pre-induction phase ( $84 \%$ vs. $28 \%$ ), especially during tracheal intubation and extubation, when there were $72 \%$ arrhythmias being $43 \%$ ventricular arrhythmias, with higher incidence in patients with preexisting heart disease (60\% vs. $37 \%)$. These studies, however, have not identified age brackets.

Isolated ventricular arrhythmias in asymptomatic patients or with normal ventricular function were not risk factors for perioperative cardiovascular complications. However, complex and frequent ventricular arrhythmias with abnormal ventricular function were observed in the perioperative period, especially in patients with history of heart disease and have led to major complications and cardiac death. Patients with history of heart disease, abnormal ventricular function and frequent and complex ventricular arrhythmias had a higher number of complications and higher mortality rate ${ }^{35}$. In our study, VPC was present in group TUP versus control in: $76.2 \%$ vs. $81.2 \%$ in the preoperative, $80.9 \%$ vs. $68.7 \%$ in the intraoperative, and $80.9 \%$ vs. $81.2 \%$ in the postoperative periods.

Differently from Marshall et al. ${ }^{36}$ (1972) our study has shown that ventricular arrhythmias (isolated, paired and ventricular tachycardia) were not responsible for both intra and postoperative cardiovascular complications, and that in spite of the high prevalence of supraventricular and ventricular arrhythmias in this age bracket, there have been no significant differences when spinal anesthesia was used.

TUP has not increased the number of arrhythmias as compared to control group and no patient had TUP syndrome signs during the study.

Post-extracardiac surgery arrhythmias are frequent and may be triggered by other complications not inherent to heart, such as bleeding, infection, acid-base and hydroelectrolytic disturbances, and hypoxemia ${ }^{36}$. Preoperative dynamic ECG (Holter System) may be indicated when there is suspicion of silent myocardial ischemia in heart disease patients. Several studies have shown the relationship between preoperative ST depression and the frequency of ischemic events. Mangano et al. ${ }^{35}$ (1990) have shown $25 \%$ of intraoperative ischemic episodes in 200 patients. In a study involving 474 males at high risk or with known heart disease using two-channel Holter System monitoring during elective non-cardiac surgeries, two days before surgery, during surgery and two days after surgery, it has been observed that Holter-recorded myocardial ischemia before surgery has increased 9.2 times the risk for postoperative ischemia ${ }^{35}$.

The interest in perioperative myocardial ischemia monitoring reflects the possibility of using it as a factor to predict adverse cardiac results, such as acute myocardial infarction, heart failure or death. Several risk factors have been identified in selected populations ${ }^{37,38}$. However, severe adverse events (death, infarction or angina) are uncommon, even in patients at high risk. The detection of silent myocardial ischemia may help identifying this population at increased risk for cardiovascular adverse events.

Evans et al. ${ }^{39}$ (1991) have quantified hemodynamic changes during TUP using transesophageal echocardiography and have observed an increase in left ventricle afterload indicating an increase in myocardial work and oxygen demand, which may result in myocardial ischemia contributing to cardiovascular morbidity and mortality. Heart rate and systolic volume are progressively decreased during the first 30 minutes of surgery, resulting in cardiac output decrease, with significant LV afterload increase since the beginning of the procedure. These findings have shown that hemodynamic responses non-detectable by conventional monitoring methods were present during TUP. Increased LV afterload indicates increased myocardial work and oxygen demand, which may result in myocardial ischemia and contribute to increased post-TUP cardiovascular morbidity and mortality.

Dobson et al. ${ }^{40}$ (1994) have studied hemodynamic variables of patients submitted to TUP under general and spinal anesthesia and have concluded that both techniques are associated to major hemodynamic changes right after anesthetic induction, being more severe with general anesthesia. They have also observed that prostate resection period is not related to significant hemodynamic changes.

Edwards et al. ${ }^{41}$ (1995) have studied perioperative myocardial ischemia incidence and duration using outpatient ECG in 100 patients submitted to transurethral prostatectomy and randomized to general or spinal anesthesia. Total myocardial ischemia went from $18 \%$ in the preoperative period to $26 \%$ in the postoperative period. Patients with ischemic disease had a significantly higher incidence of postoperative ischemia as compared to patients without previous ischemic disease. There has been increase in postoperative ischemia incidence and duration with both anesthetic techniques, however without significant difference between them.

In a prospective randomized study, Shalev et al. ${ }^{42}$ (1999) have compared acute myocardial infarction incidence and morbidity in patients submitted to TUP, conventional prostatectomy and other non-cardiac surgeries. Post-prostatec- 
tomy AMI incidence with both techniques was higher than $6 \%$ as compared to other surgeries, where it is $2.5 \%$. There have been no statistical differences in mortality, which has been $14.4 \%$ for TUP and $8.5 \%$ for conventional prostatectomies. In our study, the incidence of ST changes for group TUP versus control was: $19 \%$ vs. $18.7 \%$ in the preoperative period, $4.8 \%$ vs. $18.7 \%$ in the intraoperative period, and $14.3 \%$ vs. $18.7 \%$ in the postoperative period without statistical differences between groups. These results are comparable to those found by Edwards et al. ${ }^{41}$ (1995).

Supraventricular and ventricular arrhythmias, and ST changes were not responsible for intra and postoperative complications in both groups. We believe that a high number of premature complexes does not counterindicate transurethral prostatectomy in elderly patients.

Our conclusion was that elderly patients have a high supraventricular and ventricular arrhythmia prevalence and that the total number of preoperative supraventricular and ventricular arrhythmias and ST changes has not been changed by transurethral prostatectomy or inguinal hernia repair in the intra and postoperative periods.

\section{REFERÊNCIAS - REFERENCES}

01. Priebe $\mathrm{HJ}$ - The aged cardiovascular risk patient. $\mathrm{Br} \mathrm{J}$ Anaesth, 2000;85:763-778

02. Oskvig RM - Special problems in the elderly. Chest, 1999;115:(Suppl5):158S-164S.

03. Costa EFA, Porto CC, Almeida JC et al - Semiologia do Idoso, em: Porto CC - Semiologia Médica. Rio de Janeiro, Guanabara Koogan, 2001;165-197.

04. Muravchick S - Anesthesia for the Elderly, em: Miller RD - Anesthesia. New York, Churchill Livingstone, 2000;2140-2156.

05. Manhães WL - O Risco e o Prognóstico na Anestesia, em: Manica J - Anestesiologia Princípios e Técnicas. Porto Alegre, Artes Médicas, 1997;80-93.

06. Shipton EA - The peri-operative care of the geriatric patient. S Afr Med J, 1983;63:855-860.

07. Haagensen R, Steen PA - Perioperative myocardial infarction. Br J Anaesth, 1988;61:24-37.

08. Cohn PF, Lawson WE - Characteristics of silent myocardial ischemia during out-of-hospital activities in asymptomatic angiographically documented coronary artery disease. Am J Cardiol, 1987;59:746-749.

09. Nademanee K, Intarachot V, Singh PN et al - Characteristics and clinical significance of silent myocardial ischemia in unstable angina. Am J Cardiol, 1986;58:26B-33B.

10. Tarhan S, Moffitt EA, Taylor WF et al - Myocardial infarctation after general anaesthesia. JAMA, 1972;220:1451-1454.

11. Steen PA, Tinker JH, Tarhan S - Myocardial reinfarctation after anesthesia and surgery. JAMA, 1978;239:2566-2570.

12. Plumlee JE, Boettner RB - Myocardial infarctation during and following anesthesia and operation. South Med J, 1972;65: 886-889.

13. Rao TL, Jacobs KH, El-Etr - Reinfarctation following anesthesia in patients with myocardial infarctation. Anesthesiology, 1983;59:499-505.

14. Friedmann AA, Grindler J - Aplicações clínicas do eletrocardiograma no idoso. Rev Soc Cardiol Estado de São Paulo, 1999;9:286-292.
15. Bertrand CA, Steiner NV, Jameson AG et al - Disturbances of cardiac rhythm during anesthesia and surgery. JAMA, 1971;216:1615-1617

16. Santos SCM, Wajgarten M, Serrok-Azul JB - Arritmias no idoso. Rev Soc Cardiol Estado de São Paulo, 1998;8:117-126.

17. Fujino M, Okada R, Arakawa K - The relationship of aging to histological changes in the conduction system of the normal human heart. Jpn Heart J, 1983;24:13-20.

18. Lakata EG - Diminished beta-adrenergic modulation of cardiovascular function in advanced age. Cardiol Clin, 1986;4:185-200.

19. Wyatt MG, Stower MJ, Smith PJ et al - Prostatectomy in the over 80 year-old. Br J Urol, 1989;64:417-419.

20. Mudd DG, Deans GT, Lee BG - Prostatectomy in a district hospital. J R Coll Surg Edinb, 1990;35:365-368.

21. Deedwania PC, Carbajal E - Silent myocardial ischaemia. A clinical perspective. Arch Intern Med, 1991;151:2373-2382.

22. Vale NB, Simonetti MPB - Farmacologia dos Anestésicos Locais, em: Imbelloni LE - Tratado de Anestesia Raquidiana. Curitiba, Posigraf, 2001;22-35.

23. Bratanow N, Atlee JL - Perioperative arrhythmia's. Seminars in Anesthesia, 1996;15:122-131.

24. Atlee JL - Perioperative Cardiac Dysrhythmias, em: Atlee JL Cardiac Dysrhythmias and Anesthesia: Mechanisms, Recognition, Management. Chicago: Year Book Medical Publishers, 1985;101-113.

25. Wajngarten M, Grupi C, Bellotti G - Frequency and significance of cardiac rhythm disturbances in healthy elderly individuals. $J$ Electrocardiol, 1990;23:171-176.

26. Shiraishi I, Takamatsu T, Minamikawa T et al - Quantitative histological analysis of human sinoatrial node during growth and aging. Circulation, 1992;85:2176-2184.

27. Tammaro AE, Ronzoni D, Bonaccorso O et al - Arrhythmia's in the elderly. Minerva Med, 1983;74:1313-1318.

28. Hashiba K - Arrhythmia's in the elderly. Nippon Ronen Igakkai Zasshi, 1989;26:101-110.

29. Wakida Y, Okamoto Y, Iwa T - Arrhythmias in centenarians. Pacing Clin Electrophysiol, 1994;17:2217-2221.

30. Waktare JE, Camm AJ - Acute treatment of atrial fibrillation why and when to maintain sinus rhythm. Am J Cardiol, 1998;81: 3C-15C.

31. Brodsky M, Wu D, Denes P et al - Arrhythmias documented by 24 hour continuous electrocardiographic monitoring in 50 male medical students without apparent heart disease. Am J Cardiol, 1977;39:390-395

32. Rasmussen V, Jensen G, Schnohr P - Premature ventricular beats in healthy adult subjects 20 to 79 years of age. Eur Heart J, 1985;6:335-341.

33. Dietz A, Walter J, Bracharz $\mathrm{H}$ et al - Cardiac arrhythmias in active elderly persons-age dependence of heart rate and arrhythmias. Z Kardiol, 1987;76:89-94.

34. Kuner J, Enescu V, Utsu F et al - Cardiac arrhythmia's during anesthesia and surgery. JAMA, 1952;150:1212-1216.

35. Mangano DT, Browner WS, Hollenberg M et al - Association of perioperative myocardial ischaemia with cardiac morbidity and mortality on men undergoing noncardiac surgery. The study of perioperative ischemia research group. N Engl J Med, 1990;323:1781-1788.

36. Marshall BE, Wyche Jr MQ - Hypoxemia during and after anesthesia. Anesthesiology, 1972;37:178-209.

37. Raby KE, Goldman L, Creager MA et al - Correlation between preoperative ischemia and major cardiac events after peripheral vascular surgery. N Engl J Med, 1989;321:1296-1300.

38. Landesberg G, Luria MH, Cotev S et al - Importance of long-duration postoperative ST-segment depression in cardiac morbidity after vascular surgery. Lancet, 1993;341:715-719.
Revista Brasileira de Anestesiologia Vol. 54, № 2, Março - Abril, 2004 
39. Evans JW, Singer M, Chapple CR et al - Haemodynamic evidence for peri-operative cardiac stress during transurethral prostatectomy. Br J Urol, 1991;67:376-380.

40. Dobson PM, Caldicott LD, Gerrish SP et al - Changes in haemodynamic variables during transuretheral resection of the prostate: comparison of general and spinal anaesthesia. $\mathrm{Br} \mathrm{J}$ Anaesth, 1994;72:267-271.

41. Edwards ND, Callaghan LC, White T et al - Perioperative myocardial ischaemia in patients undergoing transurethral surgery: a pilot study comparing general with spinal anaesthesia. $\mathrm{Br} \mathrm{J}$ Anaesth, 1995; 74:368-372.

42. Shalev M, Richter S, Kessler O et al - Long-term incidence of acute myocardial infarction after open and transurethral resection of the prostate for benign prostatic hyperplasia. J Urol, 1999;161:491-493.

\section{RESUMEN}

Mandim BLS, Achá RES, Fonseca NM, Zumpano F - Disritmias Cardíacas y Alteraciones del Segmento ST en ancianos en el Perioperatorio de Resección Transuretral de la Próstata sobre Raquianestesia. Estudio Comparativo

JUSTIFICATIVA Y OBJETIVOS: Ancianos representan 25\% del total de los pacientes quirúrgicos. Muchos pacientes con enfermedad arterial coronariana (DAC) presentan electrocardiograma (ECG) pre-operatorio normal, y alta incidencia de infarto agudo del miocárdio (IAM) silencioso en la $1^{a}$ semana de pos-operatorio. Las disritmias aumentan con la edad, siendo observadas extrasístoles supraventriculares (ESSV) y ventriculares (ESV), fibrilación atrial y disturbios de la conducción intraventricular. El objetivo de este estudio fue evaluar la prevalencia de disritmias cardíacas y de alteraciones del segmento ST en el perioperatorio por intermedio del Holter en pacientes ancianos sometidos a cirugía de resección transuretral de la próstata (RTU) y herniorrafia inguinal bajo raquianestesia.

MÉTODO: Fueron evaluados 21 pacientes con edades entre 65 y 84 años, sometidos a RTU de la próstata y 16 pacientes con edades de 65 a 86 años, sometidos a herniorrafia inguinal, bajo raquianestesia. Evaluación por el Sistema Holter en el pre-operatorio (12 horas), intra-operatorio (3 horas) y pos-operatorio (12 horas).

RESULTADOS: La prevalencia de extrasístoles supraventriculares (ESSV) entre los grupos RTU el control fue, en el pre-operatorio $85,7 \%$ vs. 93,7\%, en el intra-operatorio $85,7 \%$ vs. $81,2 \%$ y en el pos operatorio de $76,2 \%$ vs. $100 \%$. Las extrasístoles ventriculares (ESV) tuvieron prevalencia de $76,2 \%$ vs. $81,2 \%$ en el pre, $80,9 \%$ vs. $68,7 \%$ en el per y $80,9 \%$ vs. $81,2 \%$ en el pos-operatorio. La prevalencia de alteraciones del segmento ST entre los grupos RTU y control fue, no pre-operatorio $19 \%$ vs. $18,7 \%$, en el intra-operatorio $4,7 \%$ vs. $18,7 \%$ y en el pos-operatorio de $14,3 \%$ vs. $18,7 \%$, sin significancia estadística.

CONCLUSIONES: Los pacientes ancianos presentan alta prevalencia de ESSV y ESV. El número total de ESSV y ESV, y alteraciones del segmento $S T$, presentes en el período pre-operatorio, no fue alterado por la cirugía de resección transuretral de la próstata, bien como por la herniorrafia inguinal, en los períodos intra y pos-operatorio. 